\title{
Slip-Stick Vibration Suppression by Modal State Control for Traction Drive-Trains
}

\author{
Michael Fleischer $^{* a)}$ Non-member, Keiichiro Kondo** ${ }^{* *}$ Member
}

(Manuscript received Oct. 28, 2014, revised May 19, 2015)

\begin{abstract}
The increasing performance of modern electric locomotives results in slip-stick vibrations in the traction drive-train due to wheel-rail contact. This can be remedied by the introduction of a novel modal state control scheme for active oscillation damping to avoid the loss of traction force caused by passive slip readhesion control. This paper describes the basic concept of the active control scheme enhanced with anti-windup and a simple starting method that can use any standard controller. The limits of the active control scheme are pointed out and are circumvented by coupling with passive readhesion control. The resulting control scheme benefits from the advantages of both control concepts utilized with different adhesion force gradients. Finally, the predicted performance of the novel control is compared with the installed control concepts based on several test runs with a European high-performance locomotive.
\end{abstract}

Keywords: vibration suppression, state control, traction drive-train, railway vehicle

\section{Introduction}

With increasing traction force a high utilization of the tractive effort level is indispensable for modern highperformance electric locomotives. This notably applies to locomotives used for rapid tourist traffic and heavy freight service as depicted in Fig. 1. Working in such a rough application area at the limits of adhesion, the wheelset occasionally operates at a wheel-rail slip higher than the maximum achievable tractive effort value due to fluctuation of the adhesion coefficient. This is the operating range where the problematic slip-stick oscillations arise, potentially causing severe loads on the mechanical components of the drive-train.

Three methods for suppressing slip-stick vibrations were published in the late ' $90 \mathrm{~s}$ where the latter two are still stateof-the-art:

- The installed PI-slip controller was expanded by derivative action to a PID-controller ${ }^{(1)}$. However, the newly proposed controller just achieves equivalent damping compared to the already installed controller.

- A passive readhesion controller ${ }^{(2)}$ reduces the traction force in case vibrations arise. As a result, the wheelrail slip decreases and the vibrations decline. The major drawback of this method is a notable loss of traction force which is unacceptable for high-performance locomotives.

- In(3) a passive mechanical vibration absorber is mounted to the wheelset preventing slip-stick vibrations to a great extent. However, wear and ageing effects of both traction drive-train and vibration absorber prohibit a highly-efficient damping of vibrations over the whole

\footnotetext{
a) Correspondence to: fleischer@ieee. org

* Heinrich-Hertz-Str. 13

91058 Erlangen, Germany

** Chiba University

1-33, Yayoi-cho, Inage-ku, Chiba-shi, Chiba 263-8522, Japan
}

operational cycle of the drive-train. Besides that, maintenance increases due to the short lifetime of the vibration absorber.

To achieve the aim of actively damping slip-stick vibrations in traction drive-trains, the first requirement is a highly dynamic traction drive control which is easily satisfied with the latest control scheme from the collaborating railway vehicle manufacturer ${ }^{(4)}$. Additionally, an accurate and suitably reduced model is necessary that optimally reflects the characteristics of the multi-inertia traction drive-train. For this purpose, simple modal model identification is proposed, deriving the parameters of an undamped three-inertia model where both wear and ageing effects of the drive-train are considered $^{(5)}$. Based on this, an effort-minimized modal state control in the frequency domain is introduced for active vibration damping in this paper and is enhanced by windup prevention. Furthermore, a starting procedure of the active anti-vibration controller from any standard controller as well as its coupling

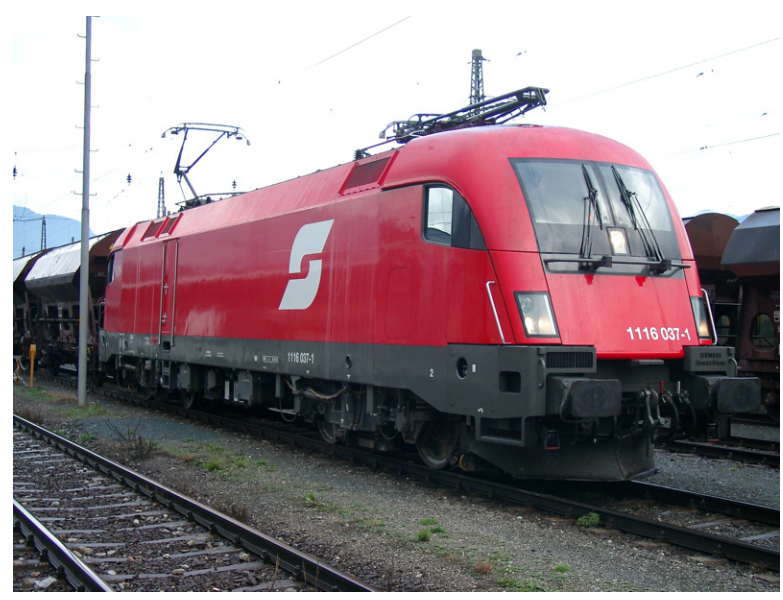

Fig. 1. European high-performance locomotive Taurus of Austrian Railways 
with the passive slip readhesion controller are discussed by means of simulation studies on a new highly-stiff traction drive-train.

\section{Highly-Stiff Traction Drive-Train}

Based on the most complex available traction drive-train in Europe, the development of an optimized drive-train for the application of an anti-vibration control scheme is presented. Figure 2(a) shows an unfolded depiction of the complex branched cardan hollow shaft traction drive-train due to its convoluted compact construction. This state-of-the-art drive-train consists of ten discrete inertias connected by torsional stiffnesses to a branched structure. Here the structure is driven by the rotor of an induction machine (1). The arrows referring to the inertia (1) indicate the known signals, the electromagnetic torque $T_{e}$ and the angular rotor speed $\omega_{r}$ which is measured by an inertia-less speed sensor, or now even sensorlessly ${ }^{(4)}$. The branching points are the gears $(\mathbf{2}, \mathbf{3}, \mathbf{8})$ with different gear transmission ratios leading to the output and braking shaft. The output shaft consists of the hollow shaft $(\mathbf{4}, \mathbf{5})$ and the wheelset $(\boldsymbol{6}, \boldsymbol{0})$ which is influenced by unknown adhesion forces $\left(z_{1}, z_{2}\right)$ due to wheel-rail contact. All torsional stiffnesses are defined by the characteristic values of steel except that connect the hollow shaft $(\mathbf{4 , 5 )}$ by rubber joints to the gear (3) and to the direct-driven wheel
(6). And finally, the braking shaft with its brake discs $(\boldsymbol{\Theta}$, (ID) is affected by the braking forces $\left(f_{1}, f_{2}\right)$. A detailed analysis of this branched structure reveals that the use of rubber couplings mainly decreases both controllability and observability of the mechanical system decoupling the slip-stick vibrations originating at the wheelset from the speed sensor of the asynchronous machine. Therefore a new optimized type of traction drive-train is investigated in Fig. 2(b). It is a major modification to the branched drive featuring highly-stiff curved-tooth couplings instead of the commonly utilized rubber couplings ${ }^{(1)}$. These drive-train modifications enormously improve the control characteristics and further reduce the maintenance costs due to the absence of rubber joint ageing. Additionally, the braking shaft is removed leading to an unbranched mechanical structure. Thus the braking forces are now directly applied to the wheelset by brake calipers. This is the most suitable fully suspended traction drive-train for anti-vibration control and it is the subject to the anti-vibration control scheme in this paper.

\section{Anti-Vibration Control Concept}

3.1 Basics First for the modal state control design, a reduced order model is identified from the new highly-stiff traction drive-train ${ }^{(5)}$. Figure 3 depicts the resulting threeinertia model, namely a motor-wheelset model. Furthermore,

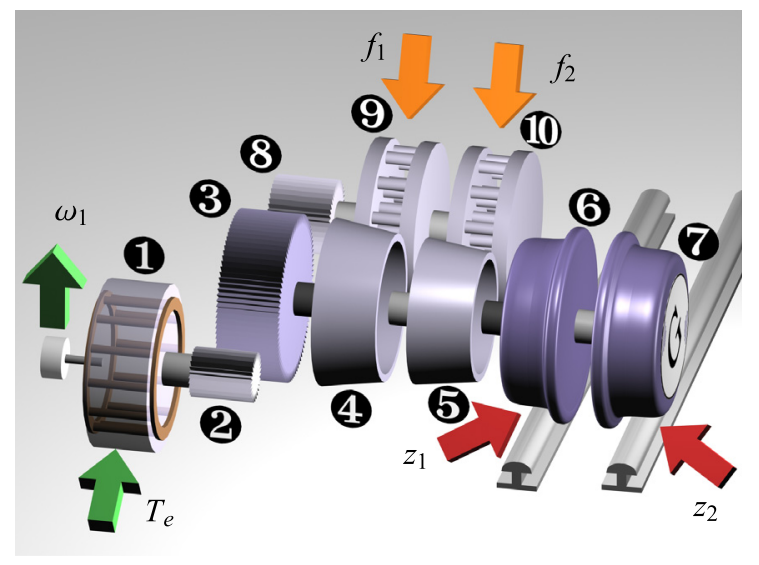

(a) Branched ten-inertia system with rubber joints

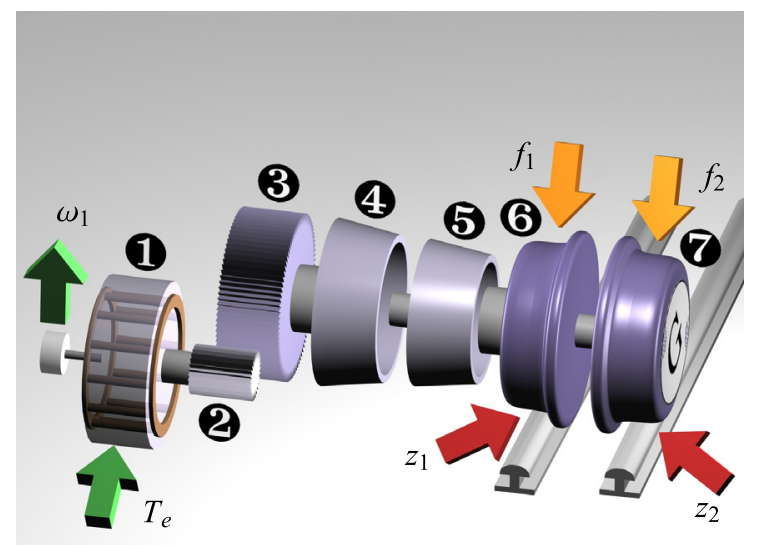

(b) Unbranched seven-inertia system with curved-tooth couplings

Fig. 2. Mechanical multi-inertia cardan hollow shaft traction drive-trains

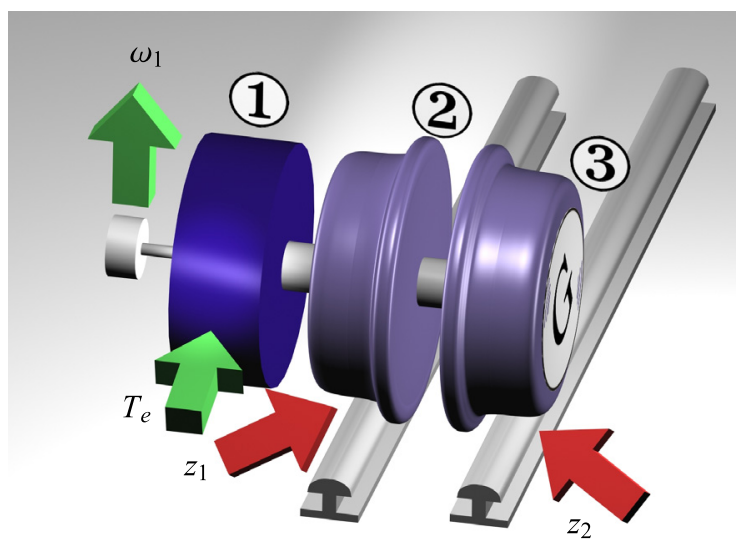

Fig. 3. Modally reduced three-inertia system for control

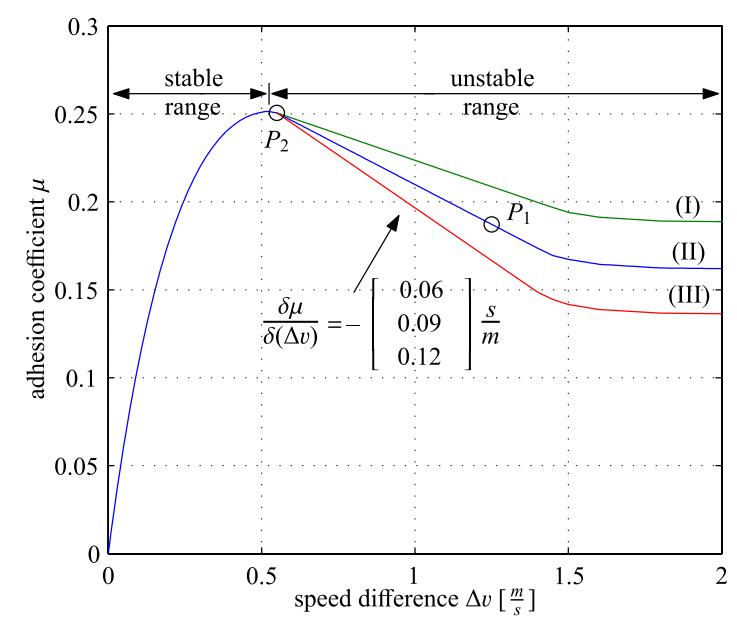

Fig. 4. Adhesive force characteristics of wheel-rail contact 
the adhesive force characteristics (I)-(III) of Fig. 4 are used in the simulation studies. The curves reflect the typical adhesion behavior of wet wheel-rail surfaces with three different adhesion force gradients at high wheel-rail slips ${ }^{(6)}$. In this slip region the operating point $P_{1}$ for wheelset conditioning is located on (II) at a wheel-rail slip of $\Delta v=1.2 \frac{\mathrm{m}}{\mathrm{s}}$. Here the gradient of the adhesion characteristic acts as an additional damping factor for the drive-train. Operating points beyond the maximum of traction coefficient, located on the negative slope, contribute a negative damping factor to the wheelset and are therefore unstable. Slip-stick oscillations consequently arise which mainly stress the wheelset shaft and increase the wheel abrasion. These oscillations are a consequence of the insufficient damping provided by the standard controller, unlike the state controller as is also proved in various applications ${ }^{(7)(8)}$. Figure 5 shows the basic concept of the proposed active anti-vibration control scheme. The observable and controllable system splits into three parts, namely the highly-stiff traction drive-train, the asynchronous machine and inverter characteristics and finally the highly dynamic torque control. For the design of the modal state controller, all nonlinearities of the electrical system including the torque control are approximated by a linear first order PT1-element and the mechanical system is represented by the modally reduced drive-train model ${ }^{(5)}$. The unknown adhesion forces are treated as disturbance inputs for the linearized system.

3.2 State Control Approach in the Time Domain

To apply state control in the time domain all system states must be available for feedback. The state of electrical system $T_{e}$ is already reconstructed by the highly-dynamic torque control using an electromagnetic torque estimator. This reduces the order of the necessary estimator by one. Hence only an estimator is required which estimates the states of the mechanical three-inertia system from the estimated electromagnetic torque $\tilde{T}_{e}$ and the rotor speed. The latter is supplied by the torque control $\tilde{\omega}_{1}$ in the lower speed range and else by the standard speed sensor $\omega_{1}$. Due to the similarity of the mechanical structures of Fig. 2(b) and Fig. 3, the adhesion forces are also taken into account for more accurate estimation results. With all state variables being supplied by the modal estimator, a conventional state controller design is carried out based on the approximated electrical system as first order lag element and on the modally reduced three-inertia system. The unknown adhesion forces are treated as disturbance inputs for the linearized system. Thus robust tracking can only be obtained by introducing integral control where an additional state variable is introduced as a negative $\omega_{1}$ output signal of an integrator. Consequently, the speed controller emerges as a simple integrator to provide a zero steady state error in the controlled variable $\omega_{1}$. To comply with the limitations imposed by the traction application, the controller order must be kept as small as possible. Using the estimated electromagnetic torque $\tilde{T}_{e}$, the order of the state controller can be reduced by one. Finally, the state control feedback gain vector $\mathbf{r}$, consisting of the vector elements $r_{1}$ to $r_{7}$, is calculated using the simple pole placement technique.

3.3 Feed Forward Control To complete the time domain concept, a classical feed-forward control is derived for the controlled variable $\omega_{1}$ from the parameters of the threeinertia system. The feed-forward controller is developed in two steps: first for the superimposed integral speed controller and second for the inner state controller $\mathbf{r}$. The best realization of the feed-forward controller is achieved if the derivation of the reference input variable $\omega_{1}{ }^{*}$, which is provided by a superimposed state-of-the-art slip controller, is included in the design. However, that would make it impossible to implement the feed-forward controller $F_{V}(s)$ for the speed controller without introducing an additional first order lag element $F_{H}(s)$ according to (9). The final stage is to develop the feed-forward controller for the inner state controller.

To this end, all feed-forward transfer functions $F_{2}(s)$, $F_{3}(s), F_{4}(s), F_{5}(s)$ and $F_{6}(s)$ are simply symbolically derived completing the state control concept design in the time

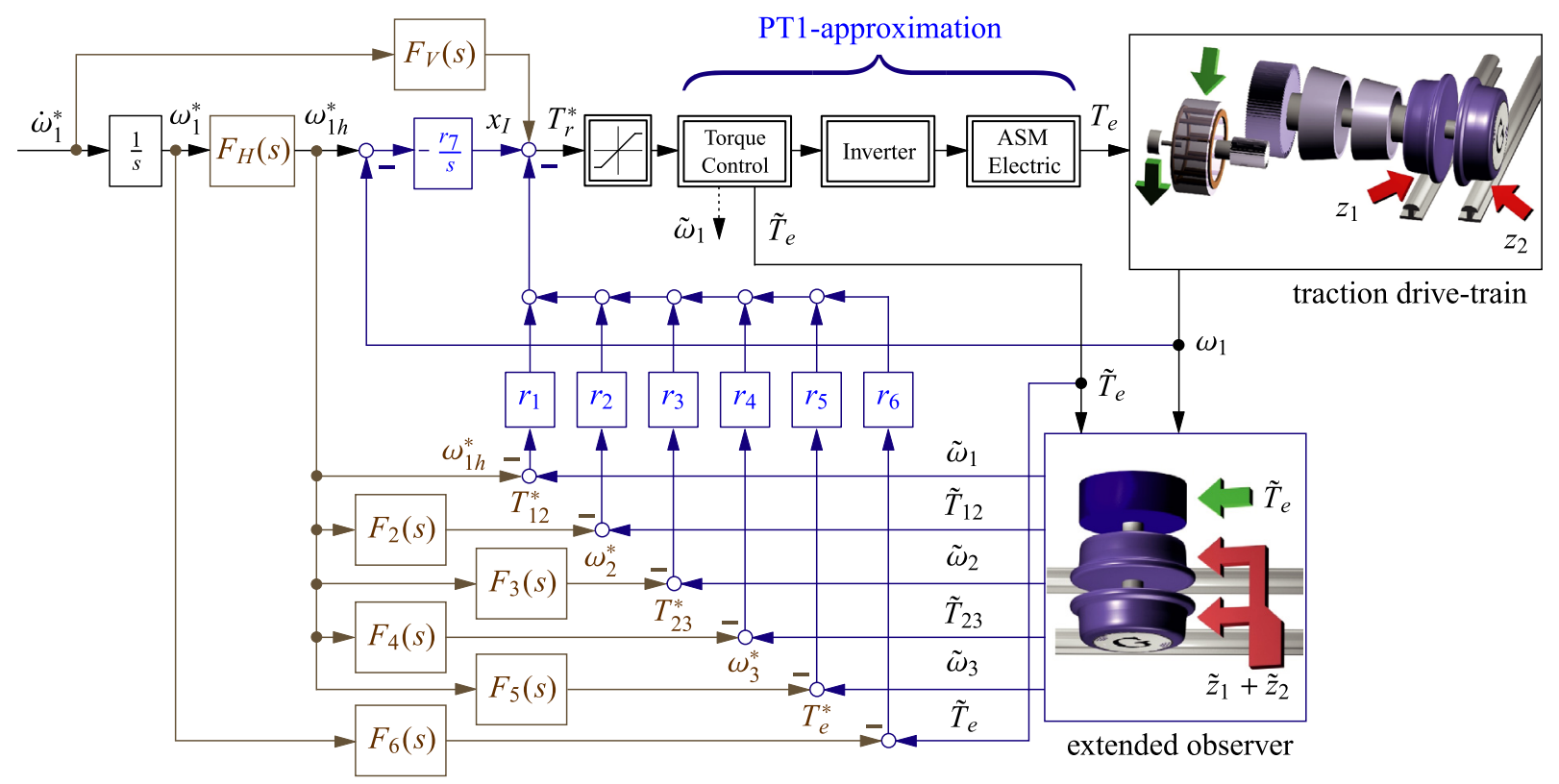

Fig. 5. Modal state control in the time domain with feed-forward control 
domain. Various simulations reveal excellent results of the feed-forward controller for the angular frequencies. By contrast, the torque feed-forwards exhibit a considerable difference corresponding to the unknown adhesion forces and thus can be neglected for further considerations.

3.4 State Control Approach in the Frequency Domain

As already mentioned, the computation time limitations of the control concept are crucial for railway applications. Thus the modal feedback controller is directly designed in the frequency domain in Fig. 6 based on the gained knowledge from the time domain approach. The denominator of the feedback controller is already fixed by the desired characteristic observer polynomial $\tilde{\Delta}(s)$ whereas the nominator polynomials $N_{\Omega}(s)$ and $N_{T}(s)$ have to be computed to assure the desired closed-loop dynamics. Finally the feed-forward of the reference speed $\omega_{1}^{*}$ reduces the action of the feedback controller. So far the effects of the system input constraint, namely the reference torque saturation, are not considered. Due to the saturating nonlinearity, undesired oscillations are triggered in the anti-vibration control scheme of Fig. 6. This effect is wellknown in control theory as windup and it has to be removed for the practical application. Here two different phenomena have to be considered, namely the controller and the plant windup. Both are discussed in the sequel with the main focus on the controller windup.

3.5 Windup Prevention Strategies Increasing the closed-loop dynamics beyond certain structurally dependent limits, plant windup occurs. Remedy is found either in the design of an additional dynamic network or in an appropriate reduction of the closed-loop dynamics. To comply with the application limitations, namely an effort-minimized control structure, an upper limit for the closed-loop dynamics is introduced using a simple design-aid for plant windup prevention $^{(10)}$. Additionally, a controller windup can be triggered both in the speed and in the feedback controller. It is due to unstable controller dynamics and is easily removed by a structural modification in the controller realization. The observer-based control structure of Fig. 6 naturally provides controller windup prevention by feedback of the saturated
Table 1. Speed controller with nonlinearity

\begin{tabular}{l|l} 
Nonlinearity & Windup prevention results \\
\hline Linear & $\frac{k_{p} s+k_{I}}{s+\delta_{1}} \frac{s+\delta_{1}}{s}$ \\
Saturation & $\frac{k_{p} s+k_{I}}{s+\delta_{1}}$ is stable
\end{tabular}

plant input, namely the reconstructed state $\tilde{T}_{e}$ of the electrical system. On the other hand, a high utilization of tractive effort causes reference torque saturation, leading to an integral windup in the speed controller. In the standard control concept shown in Fig. 7(a), windup prevention is achieved by cutting off the integral part of the speed controller in the event of control signal saturation. In order to obtain joint windup prevention for both speed and feedback controllers, the classic windup prevention is modified. Windup prevention is achieved in the speed controller by introducing a stable controller pole at $-\delta_{1}$. To substitute this pole $-\delta_{1}$ by integral action in the linear case, an appropriate first order element is arranged around the nonlinearity. This modified controller is a PI-controller in the linear case and exhibits no integral windup when saturation becomes active. Figure 7(b) shows this modified anti-windup structure and the windup prevention outcome is given in Table 1. The application of this structure to the anti-vibration control scheme of Fig. 6 concludes the windup prevention, where $k_{p}=0$ and $k_{I}=-r_{7}$ are applied.

3.6 Starting of Active Anti-Vibration Controller If the active anti-vibration control is the primary control scheme on the vehicle, the standard control concept has to be integrated as a fallback system. Consequently, the starting of the active anti-vibration controller can also be carried out from the standard controller during operation. In the event of unexpected system states at the first attempt at starting, the conventional PI-speed controller with its original closed-loop dynamics assures operational functionality of the vehicle. Using the proposed PI-controller modification, both control concepts can easily be coupled. Thus both speed controllers share the same windup prevention structure, as indicated by the dashed box in Fig. 7(b).

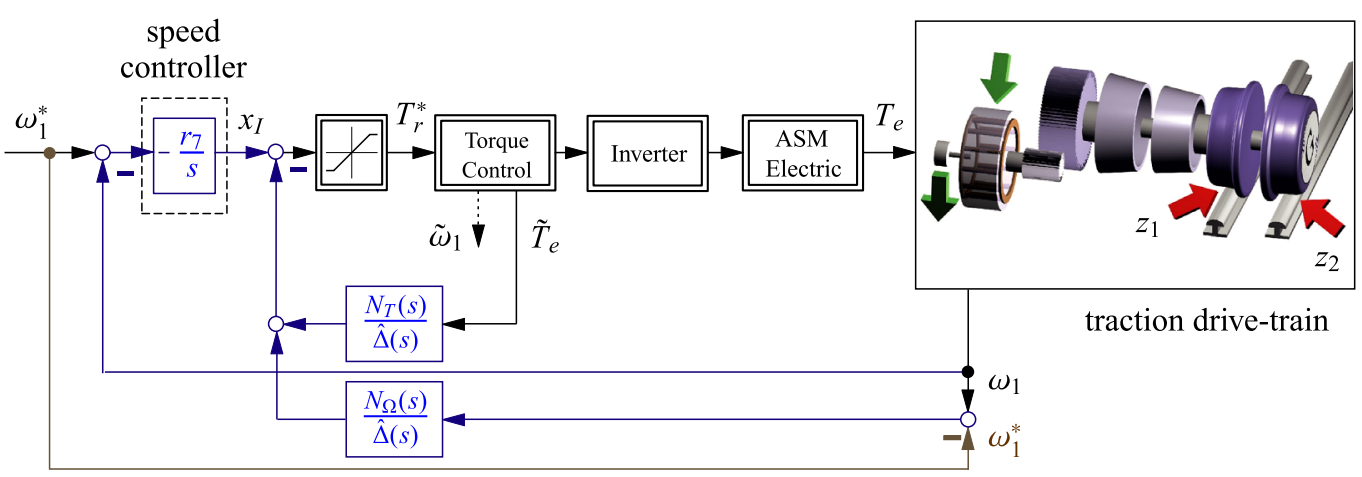

Fig. 6. Active anti-vibration control by modal state controller

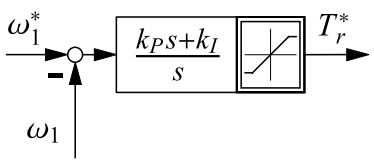

(a) classic

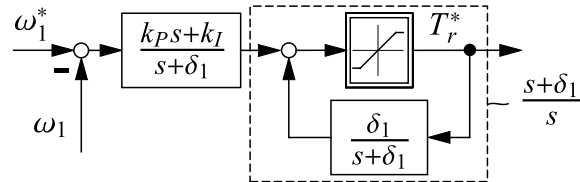

(b) modified

Fig. 7. Speed controller with anti-windup prevention schemes 
The modified control scheme shown in Fig. 8 provides effort-minimized joint windup prevention for both controllers. Compared with Fig. 6, it contains an additional transfer function in the feedback path compensating the dynamic behavior of the shared speed controller. The transition structure simply consists of a sliding factor $\kappa$. Thus the two control concepts, namely the standard controller $(\kappa=0)$ and the active anti-vibration controller $(\kappa=1)$, are the two limit values of this sliding factor. To facilitate applicability, the design of the two control concepts is decoupled. Hence the feedback controller design is independent of the parameters of the standard speed controller and therefore remains unchanged. To achieve this, the output of the standard speed controller has to be compensated when $\kappa=1$. Feeding it additively to the speed controller output of the anti-vibration controller solves this problem in a simple way. Consequently, the antivibration scheme is applicable with any standard speed controller. For control design, the first adjusting control loop is the standard controller where the dynamics of the windup prevention scheme are tested. The important constant $\delta_{1}$ is chosen as a stable pole between the imaginary axis and the pole of the approximated torque control. The second adjusting control loop is the anti-vibration controller.

In the sequel, the behavior of the complete control scheme is demonstrated by simulation studies with the adhesive force characteristic (II) of Fig. 4. The active anti-vibration controller is appropriately designed to damp the traction drivetrain in this unstable range of the adhesion force characteristic. Besides the damping behavior, soft and hard switched transitions between both control concepts are shown in Fig. 9. Starting with the standard controller $(\kappa=0)$, the maximum of the adhesion force characteristic (II) is passed, as indicated by the electromagnetic torque $T_{e}$, and the traction drive-train reaches the desired operating point $P_{1}$ in the unstable range. For all the investigations, it operates at this constant wheelrail slip $\Delta v_{i}=1.2 \frac{\mathrm{m}}{\mathrm{s}}$ of the indirect-driven wheel. In this operating point the standard controller provides insufficient damping and therefore slip-stick oscillations occur which mainly stress the measured wheelset shaft torque $T_{67}$ (indices as in Fig. 2) with less influence to the speed of the asynchronous machine $\omega_{1}$. On the locomotive, only the estimated cardan hollow shaft torque $\tilde{T}_{12}$ and the estimated wheelset shaft torque $\tilde{T}_{23}$ (indices as in Fig. 3) based on a three-inertia model are available. At $t=0.8 \mathrm{~s}$ a slow transition to the antivibration controller is carried out, only providing full damping when $\kappa=1$ is met. Slow transitions therefore apply an unnecessary amount of stress to the wheelset shaft. Consequently, the transition speed is increased and the transition to the active anti-vibration controller is further delayed to test the damping behavior at higher vibration amplitudes. The faster transition to $\kappa=1$ at $t=2.6 \mathrm{~s}$ obviously leads to a quicker application of the full damping. The delayed transition at higher vibration amplitude, however, entails a longer duration of active damping. To investigate the effects of transition speed under the same conditions, soft $(t=1.1 \mathrm{~s})$ and hard switching $(t=4.9 \mathrm{~s})$ are compared at the same vibration amplitude. As a result, the stress in $T_{67}$ and in $T_{e}$ significantly reduces with increasing transition speed. Finally the worst case of both vibration amplitude and delayed transition is considered with hard switching. Due to extreme conditions the actuated variable $T_{e}$ instantly saturates and thus triggers windup prevention. As a result, its mean value decreases and the slip $\Delta v_{i}$ declines, temporarily operating the wheelset in the stable range of the adhesive force characteristic until the torque saturation wears off. Subsequently, active damping is effective. The saturating nonlinearity is accompanied by a loss of traction force and an undershoot of $\omega_{1}$. Both effects are well-known from the use of passive readhesion controllers.

\subsection{Coupling of Active and Passive Anti-Vibration} Control Concepts For application on the vehicle, the minimum occurring gradient $\delta \mu / \delta(\Delta v)$ of the adhesion force characteristic has to be taken into consideration. To achieve efficient damping at this highly negative gradient, the closedloop dynamics of the proposed active anti-vibration control have to be increased by a newly designed feedback controller. Higher dynamics, however, also entail an increased sensor noise amplification, producing a permanently highly oscillating variable $T_{e}$. Here, the sensor noise notably originates in the speed sensor featuring an incremental encoder with a very low resolution imposed by the robustness of traction applications. Furthermore, the increase of the closed-loop dynamics is bounded to the previously derived upper limit due to plant windup prevention. Considering both limitations, the decisive factor on the closed-loop dynamics is definitely the speed sensor noise amplification. To comply with this

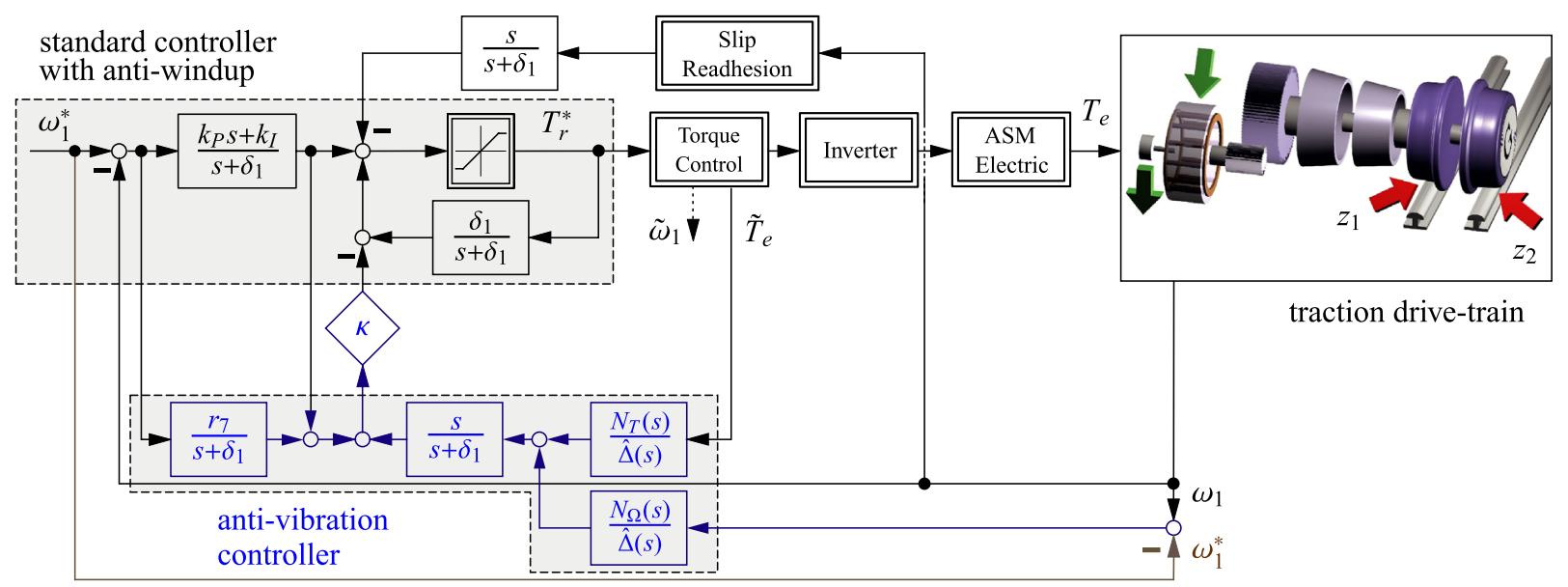

Fig. 8. Standard control concept with slip readhesion coupled with active anti-vibration control 
restriction, the active anti-vibration controller is designed for a minimum gradient of $\delta \mu / \delta(\Delta v)=-0.1 \frac{s}{m}$, capable of damping most of the slip-stick vibrations. However, at operating points with a more negative gradient, the feedback controller provides insufficient damping. Besides slip-stick vibrations, the reference torque $T_{r}^{*}$ also exhibits vibrations until saturation occurs.

A solution of this problem is found by considering stateof-the-art traction drive control systems ${ }^{(4)}$. Here, the standard speed controller only achieves sufficient damping up to a gradient of $\delta \mu / \delta(\Delta v)=-0.04 \frac{s}{m}$. At higher negative gradients a passive readhesion controller is utilized to suppress the arising slip-stick vibrations. In detail, the passive controller is tuned at the given vibration frequency. On its occurrence in $\omega_{1}$, the reference torque $T_{r}^{*}$ is reduced at the expense of traction force. Subsequently, the slip decreases and the wheelset operates in the stable range of the adhesive force characteristic where the damping of the drive is achieved. By analogy, this strategy is applied to the active anti-vibration control scheme for highly negative gradients $\delta \mu / \delta(\Delta v)>-0.1 \frac{s}{m}$. Thus the passive readhesion controller is utilized when the active anti-vibration controller fails, causing $T_{r}^{*}$ to oscillate with the vibration frequency. Finally for coupling to the anti-vibration control scheme, the behavior of the shared speed controller is also compensated here by an appropriate transfer function in the feedback branch.

In the sequel, the complete control scheme is discussed by means of simulation studies shown in Fig. 10. All three controller types, namely the standard, the active and the passive controllers, are now utilized with various gradients of the adhesive force characteristics, as given in Fig. 4. First the standard controller carries out the starting of the traction drive based upon the adhesive force characteristic (I) in the lower speed range. Subsequently, the active anti-vibration controller is gradually started in the stable range of (I), easily providing sufficient damping at the gradient of $\delta \mu / \delta(\Delta v)=$ $-0.06 \frac{s}{m}$ at operating point $P_{1}$. Due to the step-by-step decline of the gradient in the adhesive force characteristic (II) and finally in (III), the active anti-vibration controller can not cope with the highly negative gradient of $\delta \mu / \delta(\Delta v)=-0.12 \frac{\mathrm{s}}{\mathrm{m}}$ and consequently slip-stick vibrations arise. Instantly the passive readhesion controller reduces the reference torque $T_{r}^{*}$ and therefore $T_{e}$ until the active controller can cope with the gradient. Here, the new operating point $P_{2}$ is still in the unstable range of (III) with minor wheel-rail slip $\Delta v_{i}$. Using the synergy of active and passive controllers, the stable operating range of the traction drive-train is successfully expanded to $\delta \mu / \delta(\Delta v)=-0.1 \frac{s}{m}$ without any loss of traction force.

So both simulations of Fig. 9 and of Fig. 10 lead to a control scheme, where the standard controller is the primary controller and the state controller is added permanently with a low $\kappa$-value to provide some additional damping. On demand, the state controller will be fully activated by sliding $\kappa$ towards its limit value. In case of insufficient damping, the passive readhesion controller immediately reduces the traction force.

3.8 Unmodeled Dynamics and Stability Considering only an unbranched three-inertia model of Fig. 3 for a complex unbranched seven- or even for a branched ten-inertia system of Fig. 2 several vibration modes were neglected. Operating points beyond the maximum tractive effort level in Fig. 4 contribute a negative damping factor to only the first two vibration modes of any cardan hollow shaft traction drive-train and subsequently slip-stick vibrations of these vibration modes arise. The first vibration mode with the vibration frequency $\omega_{1}$ mainly twists the cardan hollow shaft $(\mathbf{4}, \mathbf{5})$ and the second vibration mode with $\omega_{2}=2.8 \omega_{1}$ the wheelset $(\boldsymbol{6}, \boldsymbol{0})$ for the most complex branched drive-train as depicted in Fig. 2(a). Higher vibration modes, such as the gear-brake shaft $(\boldsymbol{8}, \boldsymbol{9})$ with $\omega_{3}=5.2 \omega_{1}$ and as the gearhollow shaft $(3,4)$ with $\omega_{4}=6.1 \omega_{1}$ are very well damped and will not be triggered by slip-stick vibrations. Thus the suitably reduced model has just to reflect the first two vibration modes of the complex drive-train and therefore a reduced
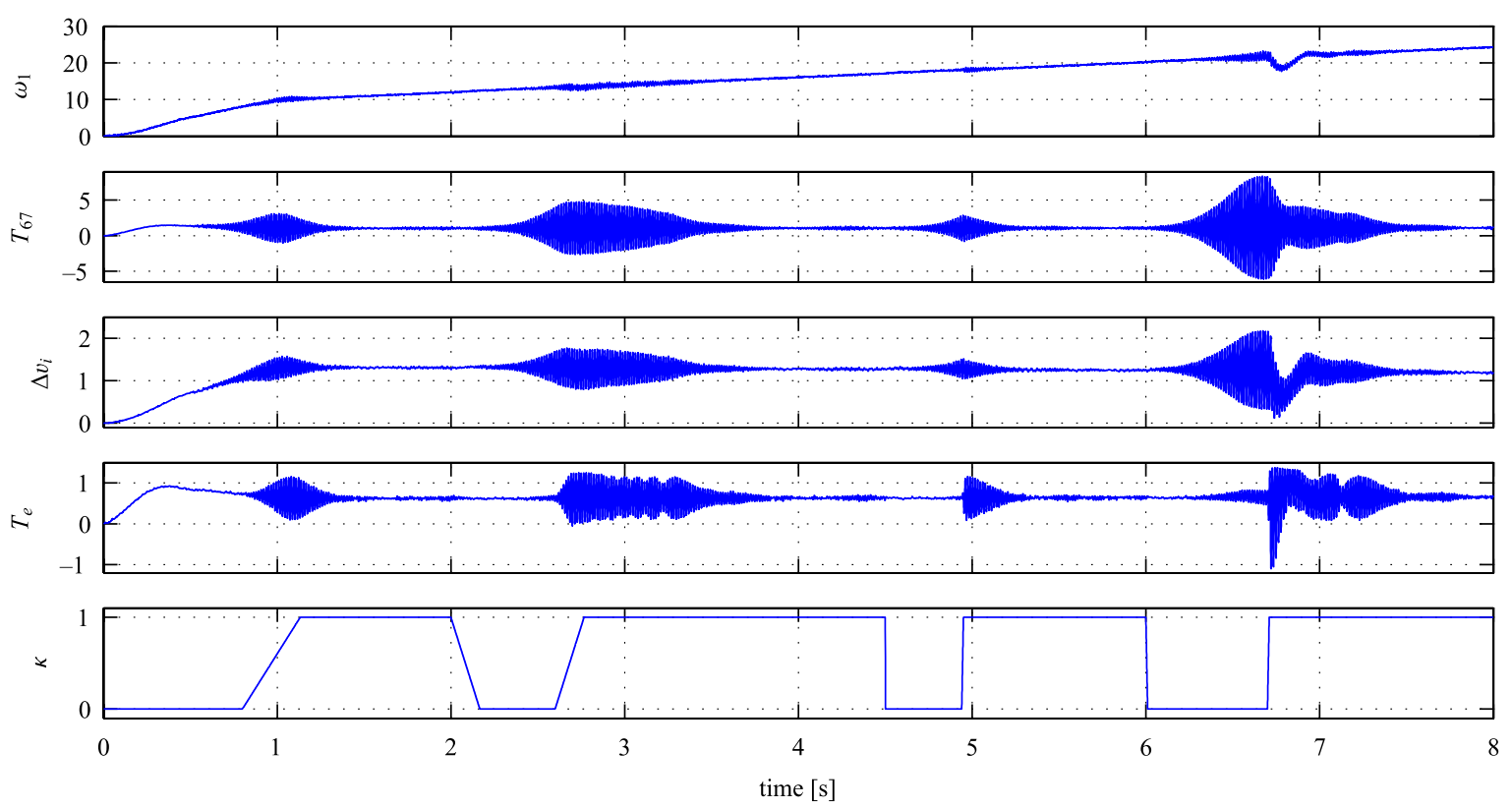

Fig. 9. Switching effects of the standard and the modal state controller $\left(\omega\left[\frac{\mathrm{rad}}{\mathrm{sec}}\right], T[p . u],. \Delta v\left[\frac{\mathrm{m}}{\mathrm{s}}\right]\right)$ 
three-inertia model is sufficient for this purpose. Further unmodeled dynamics originating from branching, wear- and ageing effects are discussed in more detail in (5). Stability of both standard and modal state controller is theoretically proven by a complex Nyquist-criterion analysis. Here, the sliding parameter $\kappa$ is used as a variable parameter transitioning both Nyquist plots. Finally, the stability of both controllers is empirically proven for any value of $\kappa$ in its range between $\kappa=0$ and $\kappa=1$ by the simulations carried out in Fig. 9 and in Fig. 10.

\section{Experimental Results}

To point out the advantages and to predict the application outcome of the proposed control scheme, the performance of the installed anti-vibration control is first investigated in several test runs with a European high-performance locomotive.

On the vehicle two control concepts are available, namely the standard control scheme and the standard control scheme coupled with a passive readhesion controller. A direct comparison of control concepts, however, is only possible if equal conditions can be achieved on consecutive test runs. Here, the decisive factor is definitely the wheel-rail contact depending on several influences, e.g. weather conditions. Besides those influencing factors, appropriate wheel-rail contact conditions have to be produced for testing control concepts in slip-stick operation. Consequently, the adhesion force characteristic of the wheel-rail contact has to exhibit a negative slope for high wheel-rail slips. Both negative slope and minimized influencing factors are achieved by watering the wheel-rail contact artificially. On the basis of those conditions several test runs of a European high-performance locomotive are conducted at a constant speed with both control concepts. All recorded data reveals the occurrence of only one slip-stick vibration frequency, namely the frequency of second vibration mode where the wheelset shaft is twisted by the wheels. Thus the wheelset shaft torque $T_{67}$ represents the best evaluation criterion for the mechanical load experienced by the drive-train for the following investigations. Furthermore, the frequency of the first vibration mode is evidently sufficiently damped by the standard controller given that it does not occur in the data. A closer look on the data reveals a difference in the adhesion characteristics in the test runs due to the stochastical behavior of the wheel-rail contact. This also applies to the unstable range of the adhesion characteristic where a wide spectrum of negative adhesion force gradients occurs leading to slipsticks vibrations. This spectrum is investigated by discretized probability distributions for the scaled oscillation amplitude of the wheelset shaft torque and for the negative adhesion force gradient which are both determined for each slip-stick vibration event. In total over three hundred events are studied for each control scheme to obtain reasonable probability distributions.

Figure 11(a) shows a comparison of two concepts for the mechanical load on the drive-train. High torque amplitudes are efficiently reduced with the use of the passive readhesion controller but can not be completely suppressed. Either the oscillations arise too quickly or the speed sensor can not reproduce the oscillations at low asynchronous machine speed due to limited sensor resolution. To validate the comparability of both torque amplitude distributions, Fig. 11(b) shows the probability distributions of the negative adhesion gradient for the conducted test runs. Those distribution curves exhibit a good match and both show the adhesion gradient of $-0.06 \frac{s}{m}$ with maximum occurrence. Furthermore, Fig. 11(b) also introduces the advantages of the proposed anti-vibration control scheme compared to the installed standard-passive concept. Active damping beyond $-0.04 \frac{\mathrm{s}}{\mathrm{m}}$ of the standard controller up to a gradient of $-0.1 \frac{\mathrm{s}}{\mathrm{m}}$ covers about $90 \%$ of all occurring negative gradients without any loss of traction force. For more negative gradients, the passive readhesion controller intervenes with use reduced by the factor of ten.

Finally, based on the analyzed data of the standard-passive test runs, the predicted outcome of the mechanical load for the novel control concept is simply obtained neglecting all oscillations where active damping applies. The resulting load spectrum in Fig. 11(a) is significantly reduced both in
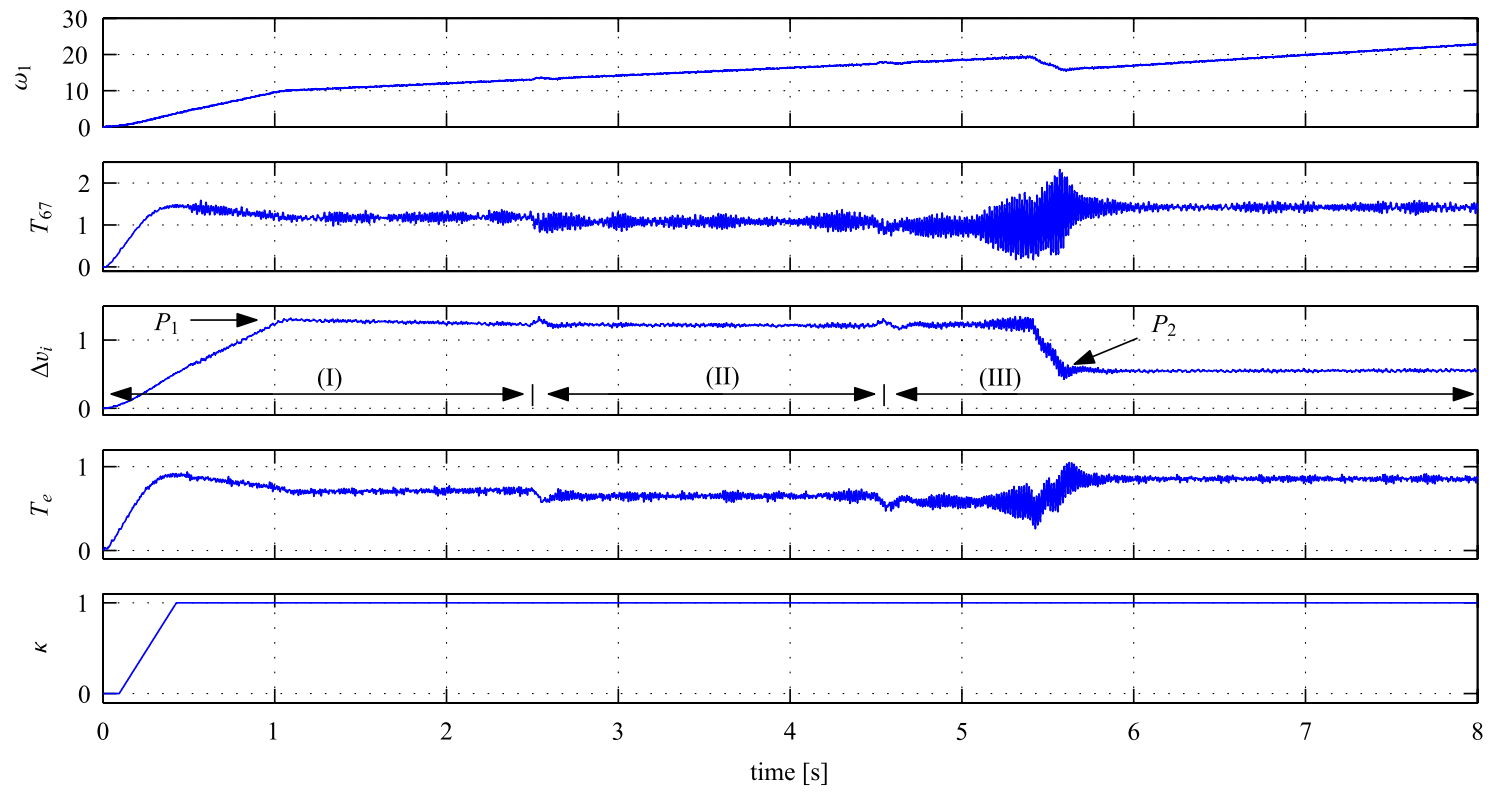

Fig. 10. Coupled active and passive anti-vibration controllers ( $\left.\omega\left[\frac{\mathrm{rad}}{\mathrm{sec}}\right], T[p . u],. \Delta v\left[\frac{\mathrm{m}}{\mathrm{s}}\right]\right)$ 


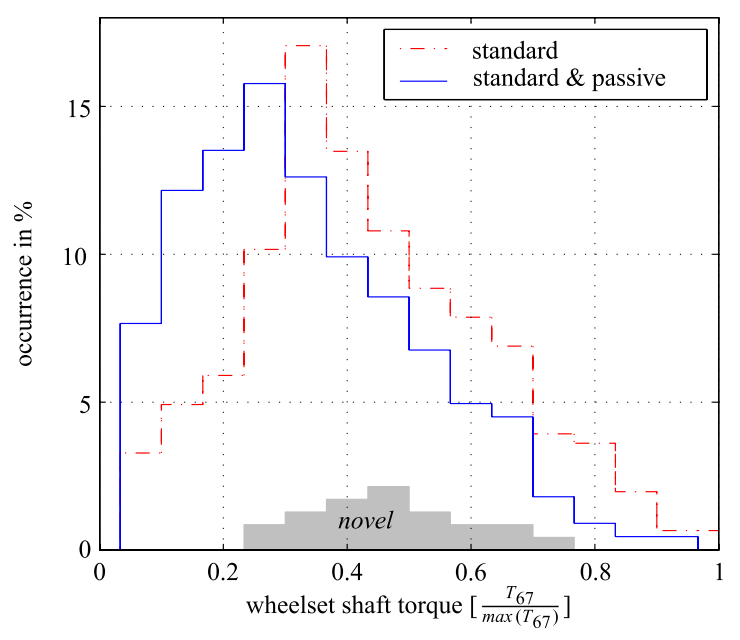

(a) Mechanical load on the wheelset shaft

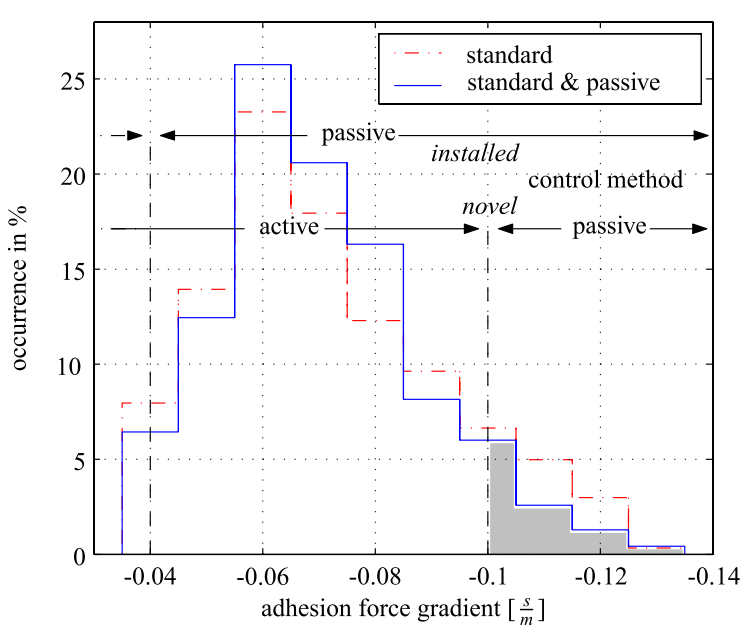

(b) Wheel-rail contact condition

Fig. 11. Probability distribution investigation into slip-stick vibrations on a European high-performance locomotive utilizing various anti-vibration control schemes

occurrence probability and maximum load for the wheelset shaft distinguishing the novel control scheme.

\section{Conclusion}

This paper presents an overall control concept for damping slip-stick vibrations in traction drive-trains. To achieve sufficient damping in the unstable range of the adhesion force characteristic, a modal feedback controller is coupled with the present standard control scheme. A simple starting procedure of this active anti-vibration controller from any standard controller is also proposed to raise its acceptance and applicability in the traction application. The resulting control scheme is enhanced by removing several windup effects. Finally, for the starting of the active control scheme, various transitions between both controllers are investigated on a new highly-stiff traction drive-train. Furthermore, the limits of the active anti-vibration controller are determined by the quality of the speed sensor signal and of the applied traction drive-train. To comply with these limitations imposed by the traction application, the active anti-vibration controller is designed to be capable of damping slip-stick vibrations up to a certain minimum gradient of adhesion force characteristic. For more negative gradients, a passive readhesion controller is additionally coupled and intervenes until the active anti-vibration controller can cope with the gradient. Using the synergy of active and passive controllers, the stable operating range of the traction drive is significantly increased without any loss of traction force. Finally, the predicted performance of the novel control is compared with the installed control concepts based on several test runs with a European high-performance locomotive.

\section{Acknowledgment}

This research project was conducted in collaboration with a well-known German railway vehicle manufacturer. The author would like to thank not only for their support, but also Japanese Railways for further investigations at the Railway Technical Research Institute in Tokyo, Japan. The purpose of this journal paper is the obtainment of my PHD degree.

\section{References}

(1) A. Jöckel: "Active Damping of Mechanical Vibrations in Drive Systems of Three-Phase AC Locomotives", (in German), in ZEVrail Glasers Annalen 125, No.5, Georg Siemens Verlag, Germany (2001)

( 2 ) H.-P. Beck and B. Engel: German Patent DE 4435775 C2, German Patent Office (1994)

( 3 ) L.A. Contreras Carranza: "Unterdrückung selbsterregter Schwingungen in Radsatzantrieben elektrischer Lokomotiven", Schwingungen in Antrieben '98, VDI Berichte No.1416 (1998)

( 4 ) G. Amler, G. Stanke, F. Sperr, M. Weidauer, and F. Hoffmann: "Highly dynamic and speed sensorless control of traction drives", in Proc. EPE '03, Toulouse, France (2003)

( 5 ) M. Fleischer: "Reduced model identification and parameter estimation for traction drive-trains", IEEJ IAS, Journal, Vol.4, No.4 (2015)

(6) A. Kawamura, M. Cao, Y. Takaoka, K. Takeuchi, T. Furuya, and K. Yoshimoto: "Measurement of tractive force in the creep region and maximum adhesion control of high speed railway systems", World Congress on Railway Research (2001)

( 7 ) K. Sugiura and Y. Hori: "Vibration suppression in 2- and 3-mass system based on the feedback of imperfect derivative of the estimated torsional torque", IEEE Trans. on Industrial Electronics, Vol.43, No.1 (1996)

( 8 ) M. Kino, N. Morita, T. Murakami, and K. Ohnishi: "A vibration suppression control of flexible manipulator by estimated reaction torque and multiple acceleration feedback", (in Japanese), Trans. IEE Japan, Vol.119-D, No.12 (1999)

(9) G. Kreisselmeier: "Two-degree-of-freedom control structure", (in German), Automatisierungstechnik 47, No.6, Oldenbourg Verlag, Germany (1999)

(10) P. Hippe and C. Wurmthaler: "Systematic closed-loop design in the presence of input saturations", Automatica 35, No.4 (1999) 
Michael Fleischer (Non-member) was awarded a Dipl.-Ing. degree (2. in electrical engineering by the Friedrich-Alexander University of Erlangen-Nürnberg, Germany, in 2002 and subsequently joined the Chair of Electrical Drives as a research associate. Funded by the Japanese Society of Promotion of Science, he worked in 2005 as a guest researcher at the Railway Technical Research Institute in Tokyo. In 2006 he entered the Trains division of Siemens as sub-project leader for customer-, R\&D- and strategy projects. In 2011 he joined German Rail as strategy project leader for both DB Energy GmbH and DB Netz AG. His main research interests are anti-vibration control and identification of flexible structures. He is a member of both IEEE and German VDE.
Keiichiro Kondo (Member) received B. S. and Dr. Eng. from the fac-

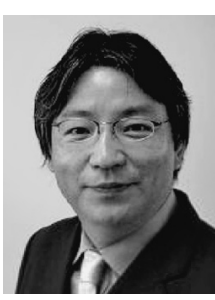
ulty of electrical engineering, department of science and technology, of Waseda University in 1991 and in 2000 respectively. He entered Railway Technical Research Institute in 1991 and had been engaged at $\mathrm{R} \& \mathrm{D}$ for power electronics applied to railway vehicle traction. Since 2007, associate professor of electrical and electronic engineering course of graduate school of Chiba University. His current research interest is power electronics, AC motor drive, energy storage devices, wireless power transmission and their applications to the railway vehicle traction. Prof. Kondo titled Dr. Eng. and Professional Engineer Japan (Mechanical Engineering, Technical Management). He is a member of both IEEE and Japanese IEEJ. 